\title{
Coupling finite element and reliability analysis through proper generalized decomposition model reduction
}

\author{
L. Gallimard, P. Vidal and O. Polit \\ LEME, Université Paris Ouest Nanterre La Défense, 50 rue de Sèvres 92410 Ville d'Avray, France
}

\begin{abstract}
SUMMARY
The FEM is the main tool used for structural analysis. When the design of the mechanical system involves uncertain parameters, a coupling of the FEM with reliability analysis algorithms allows to compute the failure probability of the system. However, this coupling leads to successive finite element analysis of parametric models involving high computational effort. Over the past years, model reduction techniques have been developed in order to reduce the computational requirements in the numerical simulation of complex models. The objective of this work is to propose an efficient methodology to compute the failure probability for a multi-material elastic structure, where the Young moduli are considered as uncertain variables. A proper generalized decomposition algorithm is developed to compute the solution of parametric multi-material model. This parametrized solution is used in conjunction with a first-order reliability method to compute the failure probability of the structure. Applications to multilayered structures in two-dimensional plane elasticity are presented. Copyright (C) 2013 John Wiley \& Sons, Ltd.
\end{abstract}

KEY WORDS: structural reliability; finite element analysis; model reduction; proper generalized decomposition; FORM approximation

\section{INTRODUCTION}

Many applications in structural analysis require to take into account stochastic properties of material, geometry, or loads. Different methods based on the probability theory have been developed to quantify the effect of data uncertainties. First-order reliability method (FORM) [1,2] is commonly used in reliability analysis. It is based on the computation of the Hasofer-Lind reliability index $\beta$ [3-7], followed by an estimation of the failure probability. In many cases, the structural response cannot be expressed explicitly, and the reliability analysis needs to be coupled with a FEM. This coupling implies difficulties, as the computation of the Hasofer-Lind reliability index involves the resolution of a constrained optimization problem and leads to multiple FEM resolutions [8]; in particular, the gradient is computed by a finite difference method, which involves two finite element computations for each parameter. In the literature, these difficulties are often circumvented by the use of response surfaces $[9,10]$. Over the past years, the development of model reduction techniques allows to consider the calculation of the complete solution set and thus to apply the optimization algorithm directly on an explicit solution. The proper generalized decomposition (PGD) has shown interesting features in the reduction model framework [11]. This type of method has been also introduced by [12] and called 'radial approximation' in the Latin method framework. It allows to decrease drastically the computational time [13]. It has been also used in the context of separation of coordinate variables in multi-dimensional PDEs [11]. For a review about the PGD and its fields of applications, the reader can refer to [14-16]. 
In this paper, a PGD algorithm is developed to compute the solution of parametric multi-materials isotropic elastic model, and the explicit solution obtained is used to compute the failure probability with a FORM approximation.

The paper is organized as follows. In Section 2, we present the basis of the reliability analysis. In Section 3, we describe the problem to be solved. Section 4 is devoted to the presentation of the application of a PGD algorithm to a parametric multi-materials elastic model. The computation of the failure probability by a FORM approximation in a PGD framework is described in Section 5. Finally, Section 6 presents an application to multilayered structures in two-dimensional plane elasticity.

\section{PRINCIPLE OF RELIABILITY ANALYSIS}

In this section, we describe the problem of reliability assessment, and we introduce the reliability index $[1,10,17]$. Structural reliability aims at computing the probability of failure of a mechanical system by accounting for uncertainties arising in a model description (geometry, material properties) or in the environmental data (prescribed displacement and external forces).

Let $\mathbf{m}$ be the vector of the model parameters on which there are some uncertainties. This vector is modeled by a random vector $\mathbf{X}$, and $f_{\mathbf{X}}(\mathbf{x})$ is the joint probability density function. The structure failure state is defined by a limit state function $G$ such that

- $G(\mathbf{x})<0$ is a failure state for the structure.

- $G(\mathbf{x})=0$ is the limit state.

- $G(\mathbf{x})>0$ is a safe state for the structure.

According to the definition of $G$, the system fails when this margin is lower or equal to 0 . The failure probability $P_{f}$ is then given by

$$
P_{f}=\int_{G(\mathbf{x}) \leqslant 0} f_{\mathbf{X}}(\mathbf{x}) \mathrm{d} \mathbf{x}
$$

The evaluation of the integral defined by Equation (1) is not easy because it represents a very small quantity and the probability density function is not usually well-known because of scarcity of statistical data. A classical way to evaluate this integral is to use the FORM [1]. An essential ingredient in the FORM in reliability analysis is to find the so-called design point. The search for this point is performed in an uncorrelated standard normal space [3]. It is therefore necessary to transform the original vector of random variables $\mathbf{X}$ into a vector $\mathbf{Y}$ defined in the space of independent standard Gaussian variables. The transformation from the variables $\mathbf{X}$ to the normalized variables $\mathbf{Y}$ is given by

$$
\mathbf{Y}=\mathcal{T}(\mathbf{X})
$$

This transformation is called an isoprobabilistic transformation $[3,18]$.

Remark

When the $x_{i}$ are jointly normal variables (the mean vector being denoted $\mathbf{M}_{x}$ and the covariance matrix $\boldsymbol{\Sigma}_{x x}$ ), the Nataf transformation [18] consists in transforming $\mathbf{X}$ to the uncorrelated standard normal space by seeking a linear transformation $\mathbf{y}=\mathbf{A x}+\mathbf{B}$ such that $\mathbf{M}_{x}=\mathbf{0}$ and $\boldsymbol{\Sigma}_{y y}=\mathbf{I}$.

The limit state function is written in the space of independent standard Gaussian variables $\mathbf{Y}$

$$
G(\mathbf{X})=G\left(\mathcal{T}^{-1}(\mathbf{Y})\right) \equiv H(\mathbf{Y})
$$

and the failure probability simplifies to

$$
P_{f}=\int_{H(\mathbf{y}) \leqslant 0} \phi(\mathbf{y}) \mathrm{d} \mathbf{y}
$$


where $\phi(\mathbf{y})$ is the $n$-dimensional standard normal density function with normal components. Firstorder reliability method analysis addresses the reliability problem in (4) by means of two key operations (see $[1,2,18,19]$ )

- First, we find the design point in the transformed independent standard normal space. The design point $P^{*}$ is the solution to the constrained optimization problem

$$
\mathbf{y}^{*}=\operatorname{argmin}\left\{\sqrt{\mathbf{y}^{T} \mathbf{y}} \mid H(\mathbf{y}) \leqslant 0\right\}
$$

where $\mathbf{y}^{*}$ is the design point and argmin denotes the argument of the minimum of a function.

- Second, we approximate the limit-state surface by the tangent hyperplane at this point and make use of the properties of the standard normal space to obtain the probability estimate.

The Hasofer-Lind reliability index, $\beta$, is defined as the minimum distance between the origin of the space $O$ and the surface of the limit state

$$
\beta=\sqrt{\left(\mathbf{y}^{*}\right)^{T} \mathbf{y}^{*}}
$$

The FORM failure probability is given by

$$
P_{F}=\Phi(-\beta)
$$

where $\Phi$ is the cumulative distribution function of a standard Gaussian variable.

\section{THE PROBLEM TO BE SOLVED}

Let us consider an elastic structure defined in a domain $\Omega$ bounded by $\partial \Omega$. The external actions on the structure are represented by a surface force density $\mathbf{T}$ defined over a subset $\Gamma_{N}$ of the boundary and a body force density $\mathbf{b}$ defined in $\Omega$. We assume that a prescribed displacement $\mathbf{u}_{d}=\mathbf{0}$ is imposed on $\Gamma_{D}=\partial \Omega-\Gamma_{N}$. The structure is made of $p$ different isotropic elastic materials occupying a domain $\Omega_{i}$, such that $\cup_{i=1}^{p} \Omega_{i}=\Omega$ and $\Omega_{i} \cap \Omega_{j}=\emptyset$ if $i \neq j$. Each material is defined by its Young's modulus $E_{i}$ and Poisson's ratio $v_{i}$. Let us consider a quantity of interest, depending on the solution of the mechanical problem $u$, denoted $S(\mathbf{u})$ (displacement of a point, Von Mises stress in a part of the structure, stress intensity factor ...) whose value must be below a critical value denoted $R$ (max displacement, yield stress, ...). In this problem, the random variables are the Young's modulus $E_{i}(i=1, \ldots, p)$ and the critical value $R$. The random vector is defined by $\mathbf{X}=\left[E_{1}, E_{2}, \ldots, E_{p}, R\right]^{T}$, and the limit state function is

$$
G(\mathbf{X})=R-S(\mathbf{u})
$$

The objective of the analysis is to compute the failure probability of the structure.

\subsection{The elastic model}

Let us denote by $\mathbf{E}$ the random vector of the material properties, $\mathbf{E}=\left[\begin{array}{ll}E_{1} & E_{2} \ldots E_{p}\end{array}\right]^{T}$. The elastic problem can be formulated as find a displacement field $\mathbf{u}(\mathbf{E})$ and a stress field $\sigma(\mathbf{E})$ defined in $\Omega$ which verify

- The kinematic constraints

$$
\mathbf{u}(\mathbf{E}) \in \mathcal{U}
$$

- The equilibrium equations

$$
\begin{aligned}
& \sigma(\mathbf{E}) \in \mathcal{S} \text { and } \forall \mathbf{u}^{*} \in \mathcal{U} \\
& -\int_{\Omega} \sigma(\mathbf{E}): \boldsymbol{\varepsilon}\left(\mathbf{u}^{*}\right) \mathrm{d} \Omega+\int_{\Omega} \mathbf{b} \cdot \mathbf{u}^{*} \mathrm{~d} \Omega+\int_{\Gamma_{N}} \mathbf{T} \cdot \mathbf{u}^{*} \mathrm{~d} \Gamma=0
\end{aligned}
$$


- The constitutive relation

$$
\sigma(\mathbf{E})=\mathbf{C}(\mathbf{E}) \boldsymbol{\varepsilon}(\mathbf{u}(\mathbf{E}))
$$

$\mathcal{U}=\left\{\mathbf{u} \mid \mathbf{u} \in\left(\mathcal{H}^{1}(\Omega)\right)^{3} ; \mathbf{u}=\mathbf{0}\right.$ on $\left.\Gamma_{D}\right\}$ is the space in which the displacement field is being sought, $\mathcal{S}=\mathcal{L}^{2}[\Omega]^{3}$ the space of the stresses, and $\boldsymbol{\varepsilon}(\mathbf{u})$ denotes the linearized deformation associated with the displacement.

\subsection{The optimization problem}

Let $\mathcal{T}$ be the isoprobabilistic transformation and $H$ the limit state function defined in the space of standardized vector $\mathbf{Y}$, from Equations (3) and (8). In this space, the failure domain $\mathcal{D}$ is defined by

$$
\mathcal{D}=\{\mathbf{y} \mid H(\mathbf{y}) \leqslant 0\}=\{\mathbf{y} \mid \mathbf{y}=\mathcal{T}(\mathbf{x}) \text { and } G(\mathbf{x})=r-S(\mathbf{u}(\mathbf{e})) \leqslant 0\}
$$

where $\mathbf{u}(\mathbf{e})$ is the solution of the elastic problem defined by Equations (9-11) for a realization $\mathbf{e}$ of the random vector $\mathbf{E}$ and $r$ is a realization of the random variable $R$.

The computation of the FORM failure probability defined by Equation (7) consists in solving the following optimization problem: find $\beta$ such that

$$
\beta=\min _{\mathbf{y} \in \mathcal{D}} \sqrt{\mathbf{y}^{T} \mathbf{y}}
$$

Usually, the optimization problem defined by Equations $(9-11,13)$ is solved by an improved version of the Hassofer-Lind-Rackwitz- Fiessler (HLRF) algorithm [4], the so-called iHLRF algorithm developed in [5-7]. This algorithm involves at each iteration the computation of solutions of Equations (9-11) by the FEM for fixed $\mathbf{y}$ (see $[5,7,19]$ for detailed studies on the convergence of the optimization problem). The iHLRF algorithm is a search algorithm that employs the search direction

$$
\mathbf{d}=\left(\alpha^{T} \mathbf{y}-\frac{H}{\|\nabla H\|}\right) \alpha-\mathbf{y} \text { with } \alpha=\frac{\nabla H}{\|\nabla H\|}
$$

which is used to built a sequence $\mathbf{y}^{(i)}(i \in \mathbb{N})$ such that

$$
\mathbf{y}^{(i+1)}=\mathbf{y}^{(i)}+\lambda \mathbf{d}
$$

The step size $\lambda$ is selected to minimize a merit function

$$
m(\mathbf{y}+\lambda \mathbf{d})=\frac{1}{2}\|\mathbf{y}\|^{2}+c|H(\mathbf{y})|
$$

where $c$ is a penalty parameter satisfying the condition $c \geqslant\|\mathbf{y}\| /\|\nabla H\|$ at each step.

\section{APPLICATION OF A PROPER GENERALIZED DECOMPOSITION ALGORITHM TO MULTI-MATERIALS}

The proper generalized decomposition (PGD) was introduced in [11] and is based on an a priori construction of separated variables representation of the solution. The following sections are dedicated to the introduction of PGD to build a parametric solution for isotropic multi-materials analysis. We consider the problem defined by Equations (9-11) as a parametrized problem where the Young's modulus in each domain $\Omega_{i}$ is in a bounded interval $\mathcal{I}_{i}=\left[e_{m}^{i} ; e_{M}^{i}\right]$. The solution of Equations (9-11) for a point $M$ of the structure depends on the values of Young's moduli and is denoted $\mathbf{u}\left(M, e_{1}, e_{2}, \ldots e_{p}\right)$, and the stress is linked with the strain by a parametrized isotropic constitutive law $\mathbf{C}$ which is defined in each domain $\Omega_{i}$ by $\mathbf{C}=e_{i} \mathbf{C}_{1}\left(v_{i}\right)$ (where $\mathbf{C}_{1}\left(v_{i}\right)$ is the elastic tensor defined for Young's modulus equal to 1). 


\subsection{The parametrized problem}

The displacement solution $u$ is constructed as the sum of $N$ products of separated functions $(N \in \mathbb{N}$ is the order of the representation)

$$
\mathbf{u}\left(M, e_{1}, e_{2}, \ldots e_{p}\right)=\sum_{i=1}^{N} f_{1}^{i}\left(e_{1}\right) f_{2}^{i}\left(e_{2}\right) \ldots f_{p}^{i}\left(e_{p}\right) \mathbf{v}^{i}(M)
$$

where $f_{j}^{i}$ and $\mathbf{v}^{i}$ are functions that must be computed during the resolution process. The problem to be solved is written as follows: find $\mathbf{u} \in \mathcal{U}$ (space of admissible displacements) such that

$$
a\left(\mathbf{u}, \mathbf{u}^{*}\right)=b\left(\mathbf{u}^{*}\right) \forall \mathbf{u}^{*} \in \mathcal{U}
$$

with

$$
\begin{aligned}
& a\left(\mathbf{u}, \mathbf{u}^{*}\right)=\int_{\Omega \times \mathcal{I}_{1} \times \mathcal{I}_{2} \ldots \times \mathcal{I}_{p}} \mathbf{C} \boldsymbol{\varepsilon}(\mathbf{u}): \boldsymbol{\varepsilon}\left(\mathbf{u}^{*}\right) \mathrm{d} \Omega \mathrm{d} e_{1} \ldots \mathrm{d} e_{p}
\end{aligned}
$$

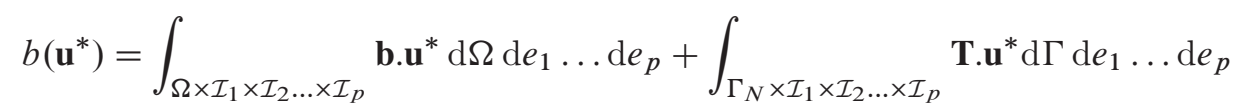

where $\Omega \times \mathcal{I}_{1} \times \mathcal{I}_{2} \ldots \times \mathcal{I}_{p}$ is the integration space associated with the geometric space domain $\Omega$ and the parameters domain $\mathcal{I}_{1} \times \mathcal{I}_{2} \ldots \times \mathcal{I}_{p}$ of the Young's moduli.

\subsection{Decomposition of the parametrized problem}

We assume that the sum of $n<N$ products of separated functions have been already computed.

$$
\mathbf{u}^{n}\left(M, e_{1}, e_{2}, \ldots e_{p}\right)=\sum_{i=1}^{n} f_{1}^{i}\left(e_{1}\right) f_{2}^{i}\left(e_{2}\right) \ldots f_{p}^{i}\left(e_{p}\right) \mathbf{v}^{i}(M)
$$

The solution at step $n+1$ is sought as the sum of $\mathbf{u}^{n}$ and a product $f_{1}^{n+1}\left(e_{1}\right) f_{2}^{n+1}\left(e_{2}\right) \ldots$ $f_{p}^{n+1}\left(e_{p}\right) \mathbf{v}^{n+1}(M)$ of unknown functions.

$$
\mathbf{u}^{n+1}\left(M, e_{1}, e_{2}, \ldots e_{p}\right)=\mathbf{u}^{n}\left(M, e_{1}, e_{2}, \ldots e_{p}\right)+f_{1}^{n+1}\left(e_{1}\right) f_{2}^{n+1}\left(e_{2}\right) \ldots f_{p}^{n+1}\left(e_{p}\right) \mathbf{v}^{n+1}(M)
$$

Let us denote by $\mathbf{f}^{n+1}$ the set of functions $\left(f_{1}^{n+1}, f_{2}^{n+1}, \ldots f_{p}^{n+1}\right)$ and by $\pi_{i}(\mathbf{f})$ and $\pi(\mathbf{f})$, the two following products

$$
\pi_{i}(\mathbf{f})=\prod_{\substack{j=1 \\ j \neq i}}^{p} f_{j} \text { and } \pi(\mathbf{f})=\prod_{j=1}^{p} f_{j}
$$

The test functions are derived from Equation (21)

$$
\mathbf{u}^{*}=\sum_{i=1}^{p} \pi_{i}\left(\mathbf{f}^{n+1}\right) f_{i}^{*} \mathbf{v}^{n+1}+\pi\left(\mathbf{f}^{n+1}\right) \mathbf{v}^{*}
$$

Introducing the trial function $\mathbf{u}$ defined by Equation (21) and the test functions $\mathbf{u}^{*}$ defined by Equation (23) into the weak form (18) leads to $p+1$ equations

- For the test functions $f_{i}^{*}, i \in\{1, \ldots, p\}$

$$
a\left(\pi\left(\mathbf{f}^{n+1}\right) \mathbf{v}^{n+1}, \pi_{i}\left(\mathbf{f}^{n+1}\right) f_{i}^{*} \mathbf{v}^{n+1}\right)=b\left(\pi_{i}\left(\mathbf{f}^{n+1}\right) \mathbf{v}^{n+1} f_{i}^{*}\right)-a\left(\mathbf{u}^{n}, \pi_{i}\left(\mathbf{f}^{n+1}\right) f_{i}^{*} \mathbf{v}^{n+1}\right) \quad \forall f_{i}^{*}
$$

- For the test function $\mathbf{v}^{*}$

$$
a\left(\pi\left(\mathbf{f}^{n+1}\right) \mathbf{v}, \pi\left(\mathbf{f}^{n+1}\right) \mathbf{v}^{*}\right)=b\left(\pi\left(\mathbf{f}^{n+1}\right) \mathbf{v}^{*}\right)-a\left(\mathbf{u}^{n}, \pi\left(\mathbf{f}^{n+1}\right) \mathbf{v}^{*}\right) \quad \forall \mathbf{v}^{*}
$$


As these equations define a coupled nonlinear problem, a nonlinear resolution strategy has to be used. The simplest strategy is the fixed point method. Starting from an initial solution $\left(\tilde{\mathbf{v}}^{(0)}, \tilde{\mathbf{f}}^{(0)}\right)$, we construct a sequence $\left(\tilde{\mathbf{v}}^{(k)}\right)_{k \geqslant 1}$ and $\left(\tilde{\mathbf{f}}^{(k)}\right)_{k \geqslant 1}$ where

- For $i \in\{1, \ldots, p\},\left(\tilde{f}_{i}^{(k)}\right)$ is defined by

$$
\begin{aligned}
& a\left(\tilde{f}_{i}^{(k)} \pi_{i}\left(\tilde{\mathbf{f}}^{(k-1)}\right) \tilde{\mathbf{v}}^{(k-1)}, \pi_{i}\left(\tilde{\mathbf{f}}^{(k-1)}\right) f_{i}^{*} \mathbf{v}\right) \\
& \quad=b\left(\pi_{i}\left(\tilde{\mathbf{f}}^{(k-1)}\right) f_{i}^{*} \tilde{\mathbf{v}}^{(k-1)}\right)-a\left(\mathbf{u}^{n}, \pi_{i}\left(\tilde{\mathbf{f}}^{(k-1)}\right) f_{i}^{*} \tilde{\mathbf{v}}^{(k-1)}\right) \forall f_{i}^{*}
\end{aligned}
$$

- And $\tilde{\mathbf{v}}^{(k)}$ is defined by

$$
a\left(\pi\left(\tilde{\mathbf{f}}^{(k)}\right) \tilde{\mathbf{v}}^{(k)}, \pi\left(\tilde{\mathbf{f}}^{(k)}\right) \mathbf{v}^{*}\right)=b\left(\pi\left(\tilde{\mathbf{f}}^{(k)}\right) \mathbf{v}^{*}\right)-a\left(\mathbf{u}^{n}, \pi\left(\tilde{\mathbf{f}}^{(k)}\right) \mathbf{v}^{*}\right) \quad \forall \mathbf{v}^{*}
$$

In practice, only few iterations of the fixed point algorithm are necessary to reach a good approximation $\left(\tilde{\mathbf{v}}^{(k)}, \tilde{\mathbf{f}}^{(k)}\right)$ of $\left(\mathbf{v}^{n+1}, \mathbf{f}^{n+1}\right)$ (see [14] for more details). The PGD leads to the algorithm given in Table I.

\subsection{Discretization}

To compute the solution of Equations (26-27), a discrete representation of the functions $\left(\mathbf{v}, f_{1}, \ldots f_{p}\right)$ must be introduced. We use a classical finite element approximation for the spatial representation in $\Omega$

$$
\mathbf{v}(M)=\mathbf{N}_{v}(M) \mathbf{q}_{v}
$$

where $\mathbf{N}_{v}(M)$ contains the finite element interpolation functions, and $\mathbf{q}_{v}$ is the vector of DOF associated with the finite element mesh in $\Omega$. And we use series expansions of functions in $\left(\mathcal{I}_{i}\right)_{1 \leqslant i \leqslant p}$

$$
f_{i}\left(e_{i}\right)=\sum_{j=-n_{1}}^{n_{2}} q_{f, i j}\left(\frac{e_{i}}{c_{i}}\right)^{j}=\mathbf{N}_{f, i}\left(e_{i}\right) \mathbf{q}_{f, i}
$$

where $n_{1}$ and $n_{2}$ determine the length of the expansion, $\mathbf{N}_{f, i}$ is the matrix of the interpolation functions, $\mathbf{q}_{f, i}$ are the DOF, and $c_{i}$ is a coefficient introduced to obtain a dimensionless function $f_{i}\left(e_{i}\right)$. In this paper, we use the bounds of the interval $\mathcal{I}_{i}$ to compute $c_{i}=0.5\left(e_{m}^{i}+e_{M}^{i}\right)$.

Table I. Proper generalized decomposition algorithm.

$$
\begin{aligned}
& \text { - for } n=1 \text { to } N_{\max } \\
& \text { Initialize } \tilde{\mathbf{v}}^{(0)} \text { and } \tilde{\mathbf{f}}_{i}^{(0)} \text { for } i \in\{1, \ldots p\} \\
& \text { for } k=1 \text { to } k_{\max } \\
& \text { for } i=1 \text { to } p \\
& \quad \text { Compute } \tilde{\mathbf{f}}_{i}^{(k)} \text { from Equation (26) } \\
& \text { end for } \\
& \text { Compute } \tilde{\mathbf{v}}^{(k)} \text { from Equation (27) } \\
& \text { Check for convergence } \\
& \text { end for } \\
& \text { Set } \mathbf{v}^{n}=\tilde{\mathbf{v}}^{(k)} \text { and } \mathbf{f}_{i}^{n}=\tilde{\mathbf{f}}_{i}^{(k)} \text { for } i \in\{1, \ldots p\} \\
& \text { Set } \mathbf{u}^{n}=\mathbf{u}^{n-1}+\pi\left(\mathbf{f}^{n}\right) \mathbf{v}^{n} \\
& \text { Check for convergence }
\end{aligned}
$$




\section{COMPUTATION OF THE FAILURE PROBABILITY}

The failure probability is computed by solving the optimization problem defined by Equation (13), with the parametrized solution obtained after $N$ iterations of the PGD, expressed as

$$
\mathbf{u}\left(M, e_{1}, e_{2}, \ldots e_{p}\right)=\sum_{i=1}^{N} f_{1}^{i}\left(e_{1}\right) f_{2}^{i}\left(e_{2}\right) \ldots f_{p}^{i}\left(e_{p}\right) \mathbf{v}^{i}(M)
$$

The isoprobabilistic transformation $\mathcal{T}$ gives the relation between the $\mathbf{x}=\left[x_{1}, \ldots x_{p+1}\right]^{T}$ and the $\mathbf{y}=\left[y_{1}, \ldots y_{p+1}\right]^{T}$. For the sake of clarity, let us assume that $x_{i}$ are independent Gaussian random variables with mean $\mu_{i}$ and standard deviation $\sigma_{i}$. Following the notations introduced in Section (3.1), the transformation in standard random variables is

$$
y_{i}=\frac{x_{i}-\mu_{i}}{\sigma_{i}} \text { for } i \in\{1, \ldots p+1\}
$$

and the inverse transformation $\mathcal{T}^{-1}$ is

$$
x_{i}=\sigma_{i} y_{i}+\mu_{i} \text { for } i \in\{1, \ldots p+1\}
$$

Each iteration of iHLRF algorithm used to solve Equation (13) requires the computation of the gradient vector $\nabla H$. It must be denoted that the parametrized representation of the solution greatly simplifies this computation because the solution depends explicitly on the random parameters. In standard finite element reliability analysis, the parametric dependency of the solution is not explicit and the computation of the gradient vector $\nabla H$ requires the computation of response surfaces $[9,10]$.

The application of the chain rule of differentiation to Equation (12) gives

$$
\nabla H=\frac{\partial r}{\partial \mathbf{y}}-\frac{\partial S}{\partial \mathbf{u}} \frac{\partial \mathbf{u}}{\partial \mathbf{e}} \frac{\partial \mathbf{e}}{\partial \mathbf{y}}
$$

in which $\frac{\partial r}{\partial \mathbf{y}}$ is computed straightforward as $\frac{\partial r}{\partial \mathbf{y}}=\left[0, \ldots, 0, \sigma_{p+1}\right]^{T} . \partial S / \partial \mathbf{u}$ is easy to compute because $S$ is usually a simple algebraic function of the parametrized response $\mathbf{u}, \partial \mathbf{e} / \partial \mathbf{y}$ is the inverse of the Jacobian matrix of the isoprobabilistic transformation. When $E_{i}$ are independent Gaussian random variables, $\partial \mathbf{e} / \partial \mathbf{y}$ is a diagonal matrix whose terms are $\sigma_{i}$ for $i \in\{1, \ldots p\}$ and 0 for $i=p+1$. The last term to be computed is the matrix $\partial \mathbf{u} / \partial \mathbf{e}$ that can be evaluated by a direct derivation of the parametrized solution (Equation (30)). The minimization of the merit function (Equation (16)) is solved in an approximate way by applying the Armijo line search [20]. Let $(s, \rho, \mu)$ a set of constants such that $s>0, \rho \in] 0,1[$, and $\mu \in] 0,1[$, the Armijo line search consists in choosing $\lambda^{(i+1)}$ to be the largest $\left(\lambda_{k}\right)_{k \in \mathbb{N}}$ such that

$$
\lambda_{k}=s \rho^{k}
$$

and

$$
m\left(\mathbf{y}^{(i)}+\lambda_{k} \mathbf{d}^{(i)}\right)-m\left(\mathbf{y}^{(i)}\right) \leqslant \lambda_{k} \mu \nabla m\left(\mathbf{y}^{(i)}\right)^{T} \mathbf{d}^{(i)}
$$

where $\nabla m\left(\mathbf{y}^{(i)}\right)$ is computed from Equation (16)

$$
\nabla m\left(\mathbf{y}^{(i)}\right)=\mathbf{y}^{i}+c \nabla H\left(\mathbf{y}^{i}\right) \operatorname{sign}\left(H\left(\mathbf{y}^{i}\right)\right)
$$

In iHLRF algorithm, the classical values chosen for $(s, \rho, \mu)$ are $(1,0.5,0.5)$. This leads to the optimization algorithm given in Table II. 
Table II. iHLRF algorithm.

- Compute the parametrized solution $\mathbf{u}\left(M, e_{1}, e_{2}, \ldots e_{p}\right)$

- Initialization $\mathbf{y}^{(0)}$

- for $i=1$ to $n_{\max }$

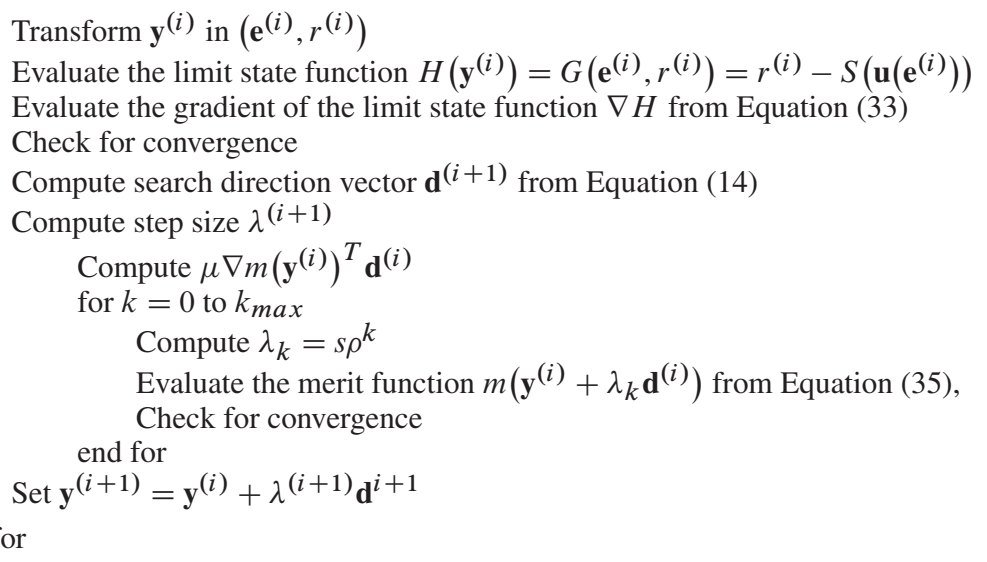

\section{NUMERICAL EXAMPLES}

The reliability analysis coupled with PGD algorithm introduced in the previous sections is tested by solving both a reliability analysis problem with a limit state function based on the maximum displacement at a given point and a problem involving a maximum Yield strength function. These two problems are solved for structures $\Omega$ made of three different materials. The spatial equations on $\Omega$ are solved with eight-node quadratic elements (Equation (28)) and the parameters of the expansions in $e$ (Equation (29)) are set to $n_{1}=1$ and $n_{2}=2$.

\subsection{Verification of the proper generalized decomposition algorithm}

To validate the PGD algorithm, we consider a structure $\Omega$ composed of three different materials as shown in Figure 1, submitted to a localized pressure $P$ in plane strain conditions. The deterministic parameters of the model are the geometry $L=200 \mathrm{~mm}, h_{1}=10 \mathrm{~mm}, h_{2}=10 \mathrm{~mm}, h_{3}=10 \mathrm{~mm}$, $L_{p}=4 \mathrm{~mm}$, the pressure $P=10 \mathrm{MPa}$, and the Poisson ratio of the materials $v_{1}=v_{2}=v_{3}=0.3$. The parametrized solution is computed on $\Omega \times \mathcal{I}_{1} \times \mathcal{I}_{2} \times \mathcal{I}_{3}$, where $\mathcal{I}_{1}=$ [126 GPa; $294 \mathrm{GPa}$ ], $\mathcal{I}_{2}=[42 \mathrm{GPa} ; 98 \mathrm{GPa}]$, and $\mathcal{I}_{3}=[108 \mathrm{GPa} ; 252 \mathrm{GPa}]$. Figure 2 shows the finite element mesh for half of the model due to the symmetry of the problem.

We define a reference solution $\mathbf{u}^{\text {ref }}$, which is the classical finite element solution computed on $\Omega \times \mathcal{I}_{1} \times \mathcal{I}_{2} \times \mathcal{I}_{3}$. A global error indicator between the reference solution $\mathbf{u}^{\text {ref }}$ and a separated variables solution $\mathbf{u}^{n}$ of order $n$ is introduced

$$
\epsilon_{n}=\sqrt{\frac{a\left(\mathbf{u}^{n}-\mathbf{u}^{r e f}, \mathbf{u}^{n}-\mathbf{u}^{r e f}\right)}{a\left(\mathbf{u}^{r e f}, \mathbf{u}^{r e f}\right)}}
$$

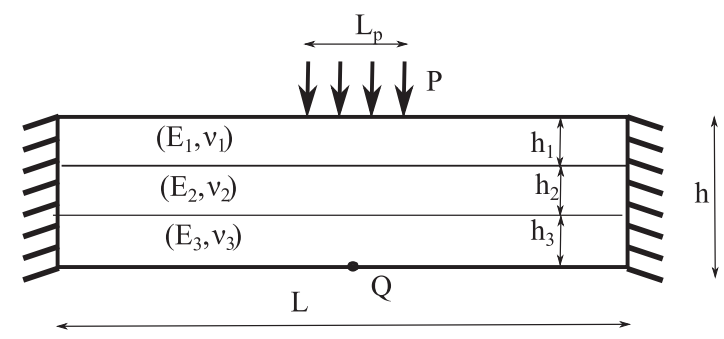

Figure 1. Example 1: multi-layered structure. 


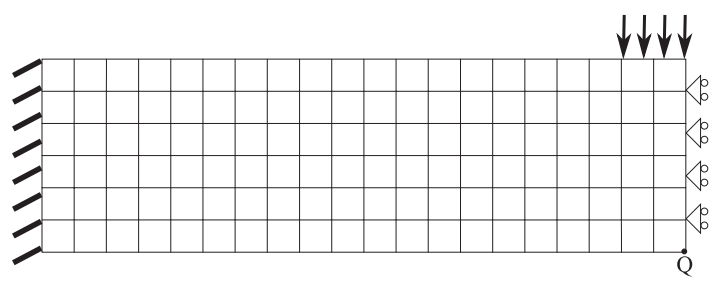

Figure 2. Mesh used for the proper generalized decomposition algorithm in example 1.

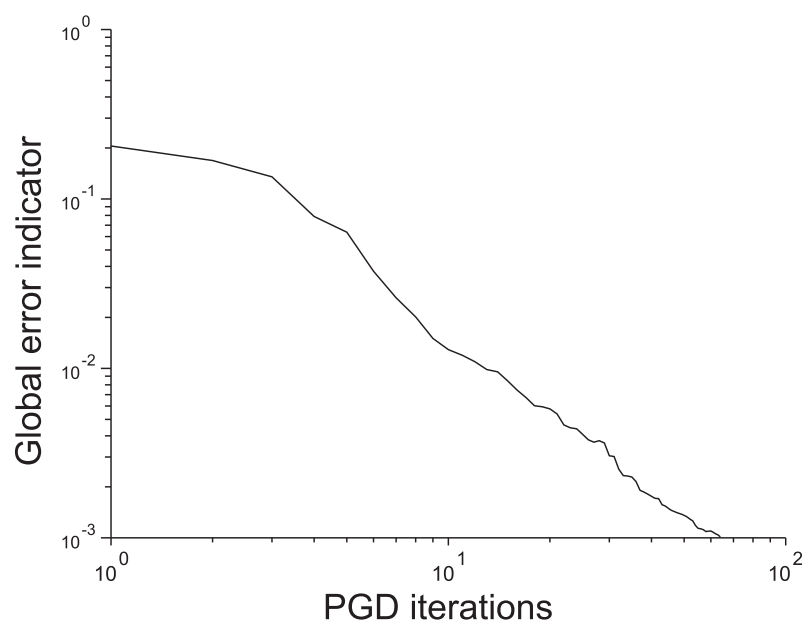

Figure 3. Convergence of the proper generalized decomposition (PGD) algorithm: evolution of $\epsilon_{n}$ with respect to the number of the PGD iterations.

The convergence of the PGD algorithm is shown in Figure 3. When a quantity of interest $S(\mathbf{u})$ is defined, an error on this quantity of interest $\epsilon_{n}^{S}$ can be computed with

$$
\epsilon_{n}^{S}=\frac{\max _{\left(e_{1}, \ldots, e_{p}\right) \in \mathcal{I}_{1} \times \mathcal{I}_{2} \times \ldots \mathcal{I}_{p}}\left|S\left(\mathbf{u}^{n}\right)-S\left(\mathbf{u}^{r e f}\right)\right|}{\max _{\left(e_{1}, \ldots, e_{p}\right) \in \mathcal{I}_{1} \times \mathcal{I}_{2} \times \ldots \mathcal{I}_{p}}\left|S\left(\mathbf{u}^{r e f}\right)\right|}
$$

Let $S(\mathbf{u})=u_{y}\left(Q, e_{1}, \ldots, e_{p}\right)$ the vertical displacement of point $Q$ (see Figure 1$)$. The convergence of the $\epsilon_{n}^{S}$ is shown in Figure 4. One can see that although the global error convergence is regular (Figure 3 ), the convergence of a local quantity as $u_{y}\left(Q, e_{1}, \ldots, e_{p}\right)$ is less regular, because the PGD algorithm minimizes only the global residual in energy. Nevertheless, 64 iterations of the PGD algorithm leads to an error approximatively equal to $1 \times 10^{-3}$ on the quantity of interest $u_{y}\left(Q, e_{1}, \ldots, e_{p}\right)$, and in the next section, $u_{y}^{64}\left(Q, e_{1}, \ldots, e_{p}\right)=S\left(\mathbf{u}^{64}\right)$ will be used as a reference value for the computation of a reference value $\beta_{\text {ref }}$ of the reliability index.

\subsection{Example 1: maximum displacement in a multilayered structure}

We consider the structure $\Omega$ described in Section 6.1 (Figures 1 and 2). The limit state function is given by

$$
G=u_{y, \max }-u_{y}(Q)<0
$$

where $u_{y}(Q)$ is the vertical displacement of point $Q$, and $u_{y, \max }$ is the maximum displacement acceptable. The random variables of the model are the Young modulus $E_{i}$ and the maximum displacement $u_{y, \max }$. They are modeled by random Gaussian independent variables and their parameters are given in Table III. The PGD algorithm described in Section 4 is used to compute a parametrized solution on $\Omega \times \mathcal{I}_{1} \times \mathcal{I}_{2} \times \mathcal{I}_{3}$. The bounds for $\mathcal{I}_{i}$ are chosen a priori but must 


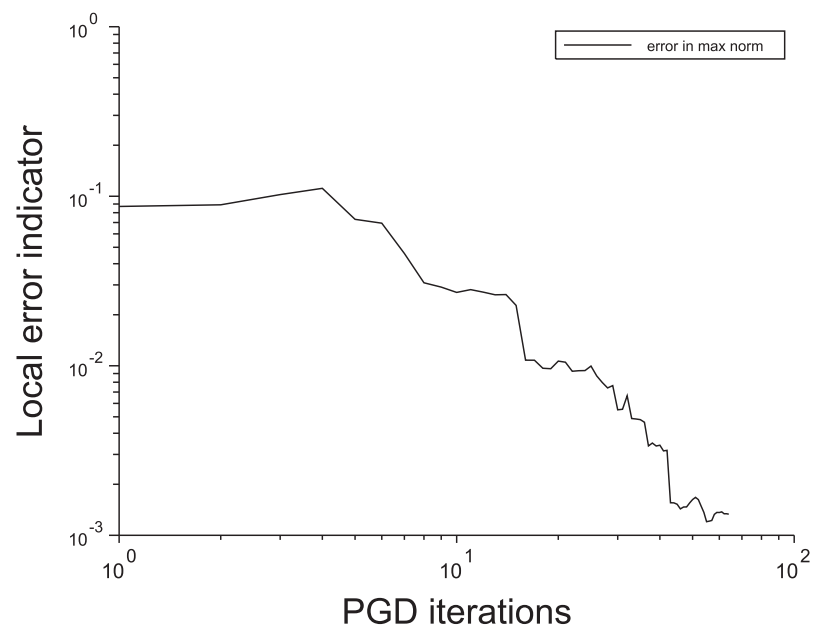

Figure 4. Convergence of $u_{y}^{n}(Q)=S\left(\mathbf{u}^{n}\right)$ : evolution of $\epsilon_{n}^{S}$ with respect to the number of the proper generalized decomposition (PGD) iterations.

Table III. Example 1.

\begin{tabular}{lccc}
\hline Variable & Probability distribution & Mean & Standard deviation \\
\hline$E_{1}(\mathrm{MPa})$ & Normal & $210 \times 10^{3}$ & $21 \times 10^{3}$ \\
$E_{2}(\mathrm{MPa})$ & Normal & $70 \times 10^{3}$ & $7 \times 10^{3}$ \\
$E_{3}(\mathrm{MPa})$ & Normal & $180 \times 10^{3}$ & $18 \times 10^{3}$ \\
$u_{y, \max }(\mathrm{mm})$ & Normal & 0.1 & 0.01 \\
\hline
\end{tabular}

be sufficiently broad to include the coordinates of the design point. Here, the bounds chosen to define $\mathcal{I}_{i}$ are the same with the one defined in Section 6.1 (i.e., $\mathcal{I}_{1}=[126 \mathrm{GPa} ; 294 \mathrm{GPa}], \mathcal{I}_{2}=$ [42 GPa;98 GPa], and $\mathcal{I}_{3}=[108 \mathrm{GPa} ; 252 \mathrm{GPa}]$ ). The expression of the vertical displacement at point $Q$ is given by

$$
u_{y}^{N}\left(Q, e_{1}, e_{2}, e_{3}\right)=\sum_{i=1}^{N}\left(\prod_{j=1}^{3} f_{j}^{i}\left(e_{j}\right)\right) v^{i}(Q)
$$

The reliability analysis is performed for different values of the order of the PGD solution, and the results are shown in Figure 5. The parametrized displacement field obtained with the first four iterations of the PGD leads to a reliability index very close to the converged value. This behavior will not be seen again on the example presented in Section 6.3. The observation of Figure 5 suggests that 12 iterations of the PGD will be sufficient to obtain a parametrized displacement field leading to a stable estimate of the reliability index. Figure 6 illustrates the convergence of the iHLRF algorithm when $N=12$. It can be seen that four iterations of the iHLRF algorithm lead to a converged solution $\beta=4.728$. Following Equation (7), the associated failure probability is $P_{F}=1.134 \times 10^{-6}$. The coordinates of the design points (i.e., $\mathbf{y}^{*}=\operatorname{argmin}_{\mathbf{y} \in \mathcal{D}} \sqrt{\mathbf{y}^{T} \mathbf{y}}$ ) are given in Table IV and are inside the bounds defined for the PGD analysis.

\section{Remark}

It must be denoted that once the parametrized finite element solution has been calculated by the PGD algorithm, no new finite element solutions need to be computed in order to perform the reliability analysis.

To obtain a reference value for $\beta$, we approximate the loading effect obtained from the reference solution $S(\mathbf{u})=u_{y}\left(Q, e_{1}, e_{2}, e_{3}\right) \approx u_{y}^{N}\left(Q, e_{1}, e_{2}, e_{3}\right)$ computed by Equation (40) with $N=64$. 


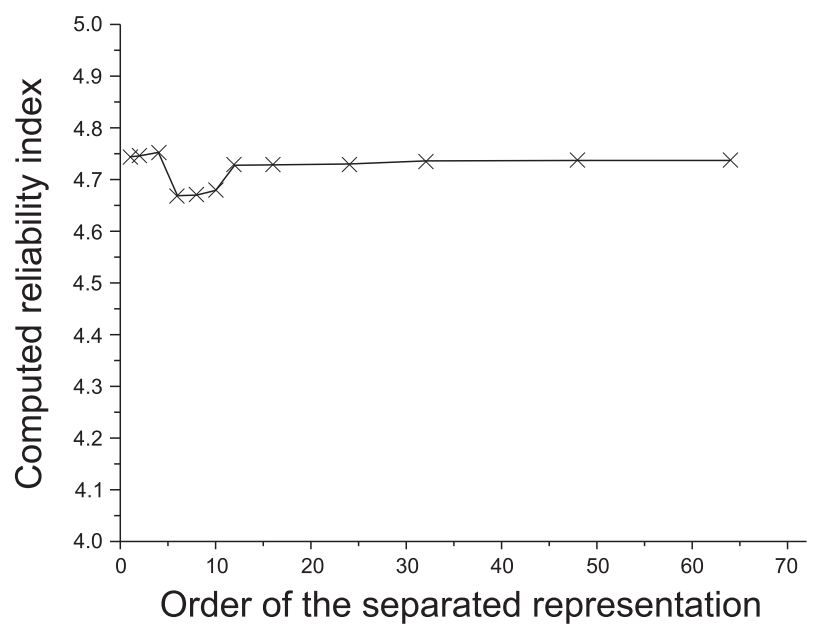

Figure 5. Convergence of the computation of $\beta$ as a function of the order of the separated representation.

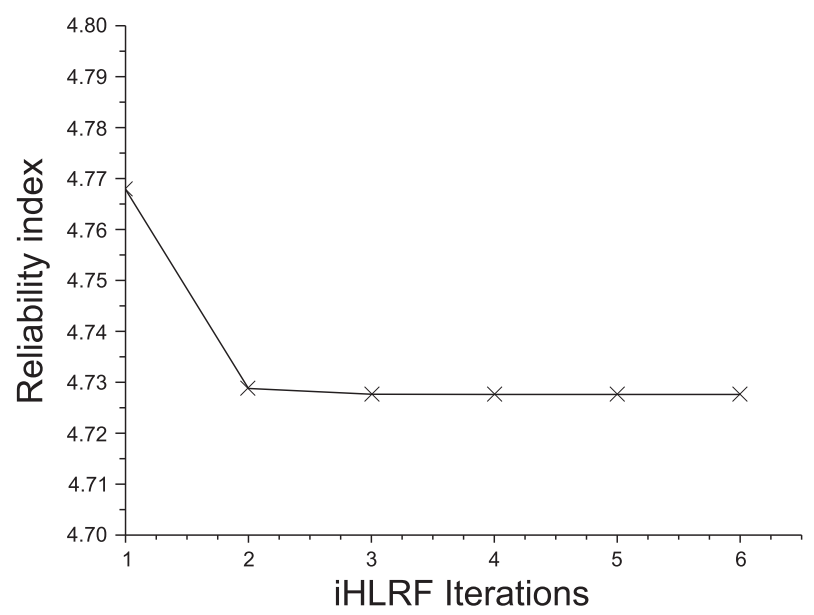

Figure 6. Convergence of the reliability during the iHLRF algorithm.

Table IV. Example 1: design point.

\begin{tabular}{lcccc}
\hline Standard Gaussian space & $y_{1}^{*}$ & $y_{2}^{*}$ & $y_{3}^{*}$ & $y_{4}^{*}$ \\
\hline \multirow{2}{*}{ Physical space } & -1.00 & -0.70 & -1.06 & -4.44 \\
& $e_{1}^{*}(\mathrm{MPa})$ & $e_{2}^{*}(\mathrm{MPa})$ & $e_{3}^{*}(\mathrm{MPa})$ & $u_{y, \text { max }}^{*}(\mathrm{~mm})$ \\
& $189 \times 10^{3}$ & $65.1 \times 10^{3}$ & $161 \times 10^{3}$ & 0.056 \\
\hline
\end{tabular}

With this approximation, it is easy to apply again the iHLRF algorithm (that converges in four iterations) and obtain a reference value for the reliability index $\beta_{\text {ref }}=4.737$ and an associated failure probability $P_{\text {ref }}=1.085 \times 10^{-6}$. We can observe that with only 12 iterations of the PGD, the accuracy of the failure probability is inferior to $5 \%$.

\subsection{Example 2: maximum Von Mises stress}

We consider a structure $\Omega$ composed of three different materials as shown in (Figure 7), submitted to a constant pressure $P$ in plane strain conditions. The limit state function is given by

$$
G=\sigma^{V M}-\frac{1}{\operatorname{mes}(\omega)} \int_{\omega} \sqrt{\sigma^{d e v}: \sigma^{d e v}} \mathrm{~d} \omega<0
$$




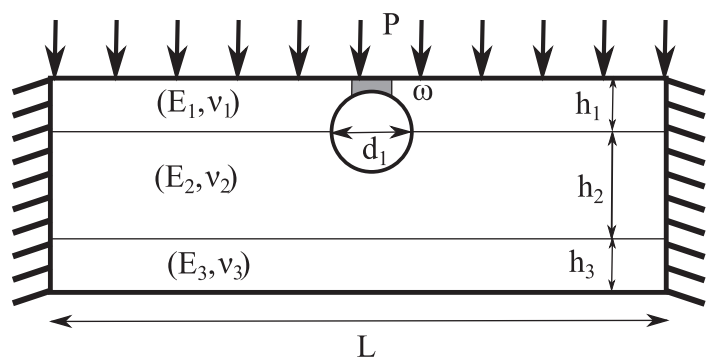

Figure 7. Example 2.

Table V. Example 2: random variables.

\begin{tabular}{lccc}
\hline Variable & Probability distribution & Mean & Standard deviation \\
\hline$E_{1}(\mathrm{MPa})$ & Normal & $180 \times 10^{3}$ & $18 \times 10^{3}$ \\
$E_{2}(\mathrm{MPa})$ & Normal & $70 \times 10^{3}$ & $7 \times 10^{3}$ \\
$E_{3}(\mathrm{MPa})$ & Normal & $210 \times 10^{3}$ & $21 \times 10^{3}$ \\
$\sigma^{V M}(\mathrm{MPa})$ & Normal & 150 & 15 \\
\hline
\end{tabular}

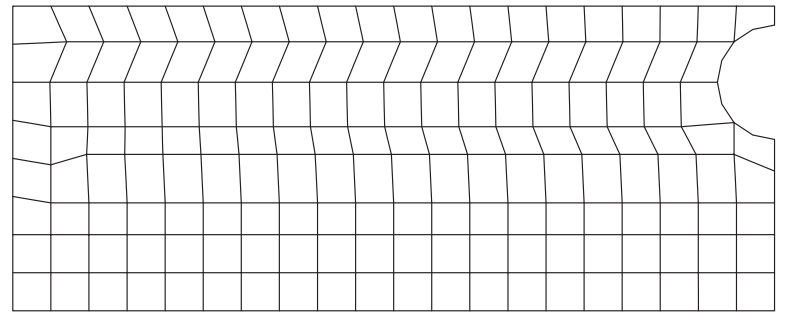

Figure 8. Mesh used for the proper generalized decomposition algorithm in example 2.

where $\omega$ is a small part of the structure described in Figure 7, $\operatorname{mes}(\omega)$ is the area of domain $\omega, \sigma^{\text {dev }}$ is the stress deviator tensor, and $\sigma^{V M}$ is the yield strength of the material in $\omega$. The deterministic parameters of the model are the geometry $L=200 \mathrm{~mm}, h_{1}=10 \mathrm{~mm}, h_{2}=20 \mathrm{~mm}, h_{3}=10 \mathrm{~mm}$, $d_{1}=15 \mathrm{~mm}$, the pressure $P=10 \mathrm{MPa}$, and the Poisson ratio of the materials $v_{1}=v_{2}=v_{3}=0.3$. The random variables of the model are the Young Modulus $E_{i}$ and the yield strength $\sigma^{V M}$, they are modeled by random Gaussian independent variables and are given in Table V. The parametrized solution is computed on $\Omega \times \mathcal{I}_{1} \times \mathcal{I}_{2} \times \mathcal{I}_{3}$, where $\mathcal{I}_{1}=[108 \mathrm{GPa} ; 252 \mathrm{GPa}], \mathcal{I}_{2}=[42 \mathrm{GPa} ; 98 \mathrm{GPa}]$, and $\mathcal{I}_{3}=[126 \mathrm{GPa} ; 294 \mathrm{GPa}]$. The mesh is shown in Figure 8 (only half of the model is meshed because of the symmetry of the problem).

For this example, the convergence of the PGD algorithm is shown in Figure 9, and the convergence of the quantity of interest is shown in Figure 10.

The reliability analysis is performed for values of the order of the PGD solution varying from 1 to 64, and the results are shown in Figure 11. On this example, a PGD order of $N=12$ is sufficient to obtain a stable evaluation of the reliability index. The computation of the failure probability is $P_{F}=1.199 \times 10^{-2}$. The coordinates of the design point are given in Table VI and are inside the bounds defined for the PGD analysis. If a PGD solution of order $N=64$ is chosen to compute a reference failure probability, the computation leads to $P_{\text {ref }}=1.191 \times 10^{-2}$. The results obtained using an approximation of order $N=12$ is very accurate. 


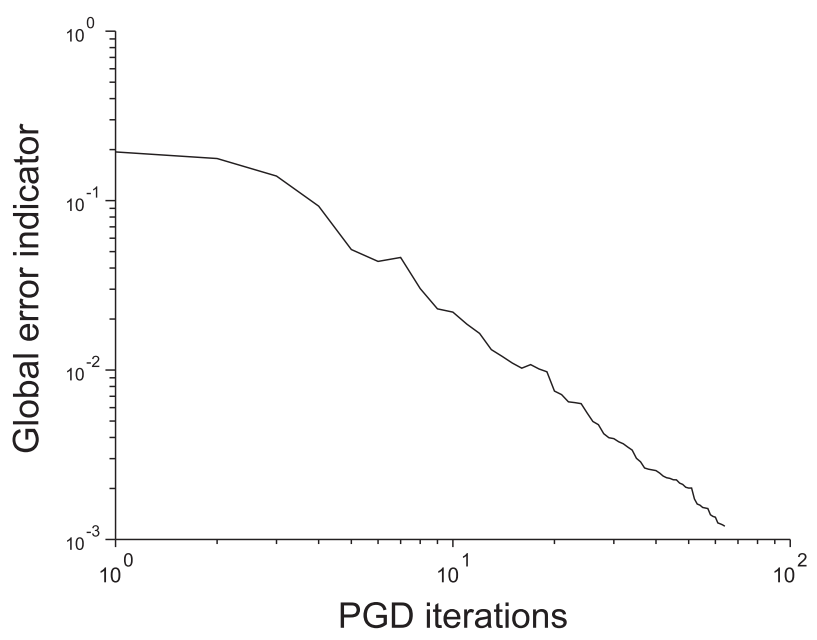

Figure 9. Example 2: evolution of $\epsilon_{n}$ with respect to the number of the proper generalized decomposition (PGD) iterations.

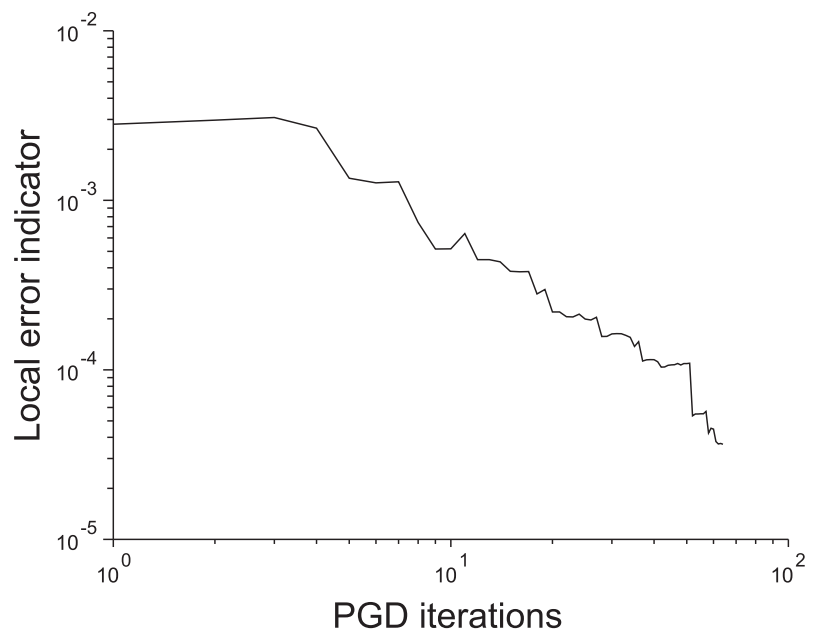

Figure 10. Example 2: evolution of $\epsilon_{n}^{S}$ with respect of the number of the proper generalized decomposition (PGD) iterations.

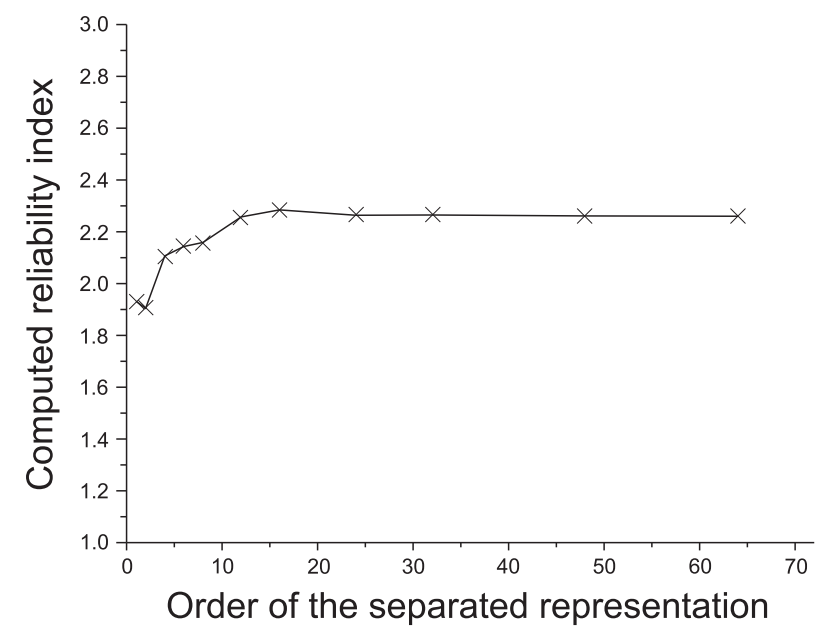

Figure 11. Convergence of the computation of $\beta$ as a function of the order of the separated representation. 
Table VI. Example 2: design point.

\begin{tabular}{lcccc}
\hline Standard Gaussian space & $y_{1}^{*}$ & $y_{2}^{*}$ & $y_{3}^{*}$ & $y_{4}^{*}$ \\
\hline & -0.218 & $-5.39 \times 10^{-2}$ & 0.267 & -2.23 \\
Physical space (MPa) & $e_{1}^{*}$ & $e_{2}^{*}$ & $e_{3}^{*}$ & $\sigma^{V M}$ \\
& $176 \times 10^{3}$ & $69.6 \times 10^{3}$ & $216 \times 10^{3}$ & 117 \\
\hline
\end{tabular}

\section{CONCLUSIONS}

A numerical methodology has been proposed to compute the failure probability of multi-materials isotropic elastic structures where the Young's moduli are uncertain parameters. The computation is carried out in two steps. In the first step, a PGD algorithm is developed allowing an a priori construction of an approximate parametrized solution of the problem to be solved. In the second step, a FORM algorithm is used to compute the failure probability of the structure. As the FORM is applied directly on an explicit parametrized solution, the computation of the derivatives which is a difficult point of the method can be carried out straightforward. The efficiency of the approach has been illustrated on examples. Future works will be devoted to the introduction of the following refined reliability methods: second-order reliability method and importance sampling method.

\section{ACKNOWLEDGEMENTS}

This research was supported by the Publishing Arts Research Council with grant number 98-1846389.

\section{REFERENCES}

1. Ditlevsen O, Madsen HO. Structural Reliability Methods. Wiley: New York, 1996.

2. Lemaire M. Fiabilité des structures. Hermes: Paris, 2005.

3. Hasofer AM, Lind NC. An exact and invariant first order reliability format. Journal of Engineering Mechanics, ASCE 1974; 100(EM1):111-121.

4. Rackwitz R, Fiessler B. Structural reliability under random load sequences. Computer and Structures 1979; 9(5):484-494.

5. Der Kiureghian A, de Stefano M. Efficients algorithms for second order reliability analysis. Journal of Engineering Mechanics 1991; 117(12):37-49.

6. Zhang Y, Der Kiureghian A. Two improved algorithms for reliability analysis. In Proceedings of the 6th IFIP WG7.5 Reliability and Optimization of Structural System, Rackwitz R, Augusti G, Borri A (eds). Chapman \& Hall, 1994; 297-304.

7. Haukaas T, Der Kiureghian A. Strategies for finding the design point in non-linear finite element reliability analysis. Probabilistic Engineering Mechanics 2006; 21:133-147.

8. Gallimard L. Errors bounds for the reliability index in finite element reliability analysis. International Journal for Numerical Methods in Engineering 2011; 87:781-994.

9. Colombi P, Faravelli L. Stochastic finite elements via response surface: fatigue crack growth problems. In Probabilistic Methods for Structural Design, Guedes Soares C (ed.). Springer: Netherlands, 1997; 313-338.

10. Pendola M, Mohamed A, Lemaire M, Hornet P. Combination of finite element and reliability methods in nonlinear fracture mechanics. Reliability Engineering and System Safety 2000; 70:15-27.

11. Ammar A, Mokdada B, Chinesta F, Keunings R. A new family of solvers for some classes of multidimensional partial differential equations encountered in kinetic theory modeling of complex fluids. Journal of Non-Newtonian Fluid Mechanics 2011; 139:153-176.

12. Ladevèze P. Nonlinear Computational Structural Mechanics - New Approaches and Non-incremental Methods of Calculation. Springer-Verlag: New York, 1999.

13. Allix O, Vidal P. A new multi-solution approach suitable for structural identification problems. Computer Methods in Applied Mechanics and Engineering 2002; 191(25-26):2727-2758.

14. Nouy A. A priori model reduction through proper generalized decomposition for solving time-dependent partial differential equations. Computer Methods in Applied Mechanics and Engineering 2010; 199(23-24):1603-1626.

15. Chinesta F, Ammar A, Leygue A, Keunings R. An overview of the proper generalized decomposition with applications in computational rheology. Journal of Non-Newtonian Fluid Mechanics 2011; 166(11):578-592.

16. Vidal P, Gallimard L, Polit O. Assessment of a composite beam finite element based on the proper generalized decomposition. Composite Structures 2012; 94:1900-1910. 
17. Mohamed A, Lemaire M, Mitteau JC, Meister E. Finite element and reliability: a method for compound variables application on a cracked heating system. Nuclear Engineering and Design 1998; 185:185-202.

18. Der Kiureghian A, Liu PL. Structural reliability under incomplete probability information. Journal of Engineering Mechanics 1986; 112(1):85-104.

19. Der Kiureghian A, Dakessian T. Multiple design points in first and second-order reliability. Structural Safety 1998; 20:37-49.

20. Shi ZJ, Shen J. Convergence of the Polak Ribière Polyak conjugate gradient method. Nonlinear Analysis: Theory, Methods and Applications 2007; 66(6):1428-1441. 